OPEN ACCESS

Edited by:

Barbara Bottazzi,

University of Milan, Italy

Reviewed by:

Angelo A. Manfredi,

Vita-Salute San Raffaele University,

Peter A. Ward

University of Michigan, United States

${ }^{*}$ Correspondence:

Benoit Brilland

benoit.brilland@chu-angers.fr

Specialty section:

This article was submitted to

Cytokines and Soluble

Mediators in Immunity,

a section of the journal

Frontiers in Immunology

Received: 05 November 2020 Accepted: 29 December 2020 Published: 16 February 2021

Citation:

Brilland $B$, Vinatier $E$, Subra J-F, Jeannin P, Augusto J-F and Delneste $Y$ (2021) Anti-Pentraxin Antibodies in Autoimmune

Diseases: Bystanders or Pathophysiological Actors?

Front. Immunol. 11:626343. doi: 10.3389/fimmu.2020.626343

\section{Anti-Pentraxin Antibodies in Autoimmune Diseases: Bystanders or Pathophysiological Actors?}

\author{
Benoit Brilland ${ }^{1,2 *}$, Emeline Vinatier ${ }^{2,3}$, Jean-François Subra ${ }^{1,2}$, Pascale Jeannin ${ }^{2,3}$, \\ Jean-François Augusto ${ }^{1,2}$ and Yves Delneste ${ }^{2,3}$ \\ ${ }^{1} \mathrm{CHU}$ Angers, Service de Néphrologie-Dialyse-Transplantation, Angers, France, ${ }^{2}$ Université d'Angers, INSERM, CRCINA, \\ Angers, France, ${ }^{3} \mathrm{CHU}$ Angers, Service d'Immunologie et Allergologie, Angers, France
}

Pentraxins are soluble innate immunity receptors involved in sensing danger molecules. They are classified as short (CRP, SAP) and long pentraxin subfamilies, including the prototypic long pentraxin PTX3. Pentraxins act mainly as bridging molecules favoring the clearance of microbes and dead cells. They are also involved in many other biological processes, such as regulation of complement activation, inflammation and tissue homeostasis. Autoantibodies directed against pentraxins have been reported in various autoimmune diseases, especially in systemic lupus erythematosus and ANCA-associated vasculitis. In this review, we review the main biological characteristics and functions of pentraxins and summarize data concerning autoantibodies directed against pentraxins in the context of autoimmune diseases and discuss their potential pathological role.

Keywords: pentraxins, autoimmunity, systemic lupus erythematosus, ANCA-associated vasculitis, antipentraxin autoantibodies

\section{INTRODUCTION}

The innate immune system is involved in many physiological processes, including antimicrobial defence, inflammation, initiation and regulation of adaptive immunity and maintenance of tissue homeostasis (1). This system has a cellular arm, composed principally of myeloid cells and innate lymphoid cells, and a humoral arm, including soluble innate immunity receptors, also known as soluble pattern recognition molecules (sPRM) (2). The innate immune system specializes in sensing of so-called "danger signals" that originate from (i) non-self, such as microbes; the microbial motifs recognized by innate cells are called pathogen-associated molecular patterns (PAMPs) or (ii) from modified (or altered) self, mainly dying cells and molecules expressed by damaged cells/tissues or accidentally released in the extracellular environment; these motifs are called damage-associated molecular patterns (DAMPs).

\footnotetext{
Abbreviations: AAV, ANCA-associated vasculitis; Ab, antibody; ANCA, anti-neutrophil cytoplasm antibody; CRP, C-reactive protein; DNA, deoxyribonucleic acid; MHC, major histocompatibility complex; MPO, myeloperoxidase; PAPS, primary antiphospholipid syndrome; PR3, proteinase 3; PTX, pentraxin; RA, rheumatoid arthritis; SAP, serum amyloid P; SLE, systemic lupus erythematosus; SLEDAI, SLE activity index; SS, Sjögren syndrome; SSA, Sjögren's-syndrome-related antigen A; SSc, systemic sclerosis.
} 
The detection of danger signals by innate immunity receptors is crucial in initiating appropriate immune responses that are fine-tuned to the motifs encountered (tolerance to self, protection against non-self). These receptors are highly conserved molecules called Pattern Recognition Receptors (PRR). PRR recognize a wide variety of ligands, including proteins, lipids, carbohydrates, and nucleic acids. In addition to innate immune cells (of myeloid and lymphoid origin), PRR are expressed by a wide variety of cell types, including endothelial cells, epithelial cells, and adaptive immune cells. Most PRR are involved in the initiation of inflammation and those expressed by professional antigen-presenting cells are critical in initiating antigen-specific immune responses.

PRR can be associated to cells (expressed at the surface or localized intracellularly) or released in the extracellular milieu (sPRM). According to their localization, cell-associated PRR can be classified in three groups (3): (i) membrane receptors, including membrane Toll-like receptors (TLR1, TLR2, TLR4, TLR5, and TLR6), scavenger receptors and C-type lectin receptors, (ii) endosomal receptors, including TLR3, TLR7, TLR8, and TLR9, and (iii) cytoplasmic receptors, including nucleotide-binding oligomerization domain (NOD)-like receptors (NLR), retinoic acid-inducible gene I (RIGI)-like receptors (RLR), and AIM2-like receptors (ALR). Membrane PRR are involved in the clearance of danger signals (i.e., phagocytosis of particulate motifs) and/or cell activation, while intracellular PRR are mainly involved in the detection of nucleic acids.

Soluble PRM act as bridging molecules, linking extracellular DAMPs and PAMPs with cell associated PRR. They include collectins, ficolins, some complement family proteins, soluble forms of membrane PRR (such as soluble scavenger receptors and C-type lectins released after shedding of the membrane forms), and by pentraxins. As their membrane counterparts, sPRM are highly conserved and bind a variety of ligands expressed by microbes and altered/modified self. Soluble PRM recognize the same molecular motifs that cellassociated PRR. They have a role in agglutination, complement activation and opsonisation (i.e., facilitating the recognition and elimination of danger motifs by phagocytes). By reference to their bridging activity, sPRM are usually viewed as ancestors of antibodies (4) and are sometimes referred to as opsonins. They also play a pivotal role in determining the nature of the immune responses induced by the motifs encountered.

A tolerance breakdown to sPRM, evidenced by the presence of autoantibodies, has been reported in different autoimmune diseases, especially autoantibodies targeting members of the pentraxin family. Such autoantibodies have been particularly reported in systemic lupus erythematosus (SLE) and anti-neutrophil cytoplasmic autoantibody (ANCA)-associated vasculitis. In this review, we present the main characteristics and functions of pentraxins and data on anti-pentraxin autoantibodies. Finally, we raise hypothesis on the potential pathological role(s) of antipentraxin autoantibodies.

\section{THE PENTRAXIN FAMILY}

\section{Structure of Pentraxins}

Pentraxins are characterized by a C-terminal "pentraxin domain" of about 200 amino acids, containing a highly conserved $\mathrm{HxCxS} / \mathrm{TWxS}$ motif (where $\mathrm{x}$ represents any amino acid) called the "pentraxin signature" $(5,6)$. They are organized in a cyclic pentameric structure $(4,7)$. The pentraxin family is divided into two subfamilies, the short and long pentraxins, depending on the length of their amino acid sequences.

Short pentraxins are characterized by a short N-terminal domain (approximatively 20 amino acids). This subfamily contains the acute phase proteins $\mathrm{C}$-reactive protein (CRP), also known as PTX1, and serum amyloid P component (SAP), also known as PTX2.

Long pentraxins are characterized by a long N-terminal domain (178 amino acids). In addition to the prototypic long pentraxin PTX3, this group contains neuronal pentraxin 1 (NP1), NP2, neuronal pentraxin receptor (NPR) and PTX4 (4, 8). Contrary to PTX3, only few data are available on these other members of the long pentraxin subfamily and will thus not be further mentioned in this review.

\section{Ligands and Immune Functions of Pentraxins}

\section{The Short Pentraxins CRP and SAP}

The short pentraxins CRP and SAP are assembled as multimers of five or ten $25 \mathrm{kDa}$ subunits of 204 and 205 amino acids, respectively. CRP and SAP are the main acute phase proteins in human and mouse, respectively. They are mainly produced by the liver in response to pro-inflammatory cytokines, such as IL-1 and IL-6. Under inflammatory situations, such as bacterial sepsis, sterile inflammation (such as gout) or massive tissue destruction (a process associated with the release of inflammatory endogenous molecules such as HSP or HMGB1), the circulating levels of CRP can rise up to 1,000-time their basal concentrations in human (3 mg/L) (9).

Short pentraxins recognize a large variety of ligands in a calcium-dependent manner. Of note, the first ligand described for CRP was the C-polysaccharide of Streptococcus pneumoniae, hence the name. SAP takes its name from the fact that it is one of the universal component of the amyloid deposits found in amyloidosis and Alzheimer's disease.

CRP and SAP promote microbe opsonisation, thus facilitating their elimination by phagocytes. The functions of short pentraxins are not limited to bacterial clearance as they can also bind many other ligands such as apoptotic cells, amyloid fibrils (10), and endogenous danger molecules, such as histones and nucleic acids released by damaged cells $(11,12)$.

Short pentraxins are potent regulators of complement pathways. They can activate the classical complement pathway by linking to the complement factor $\mathrm{Clq}$ (13) and the lectin complement pathway by binding to ficolins $(14,15)$. In contrast, they can inhibit the alternative pathway amplification loop by binding to factor $\mathrm{H}$ (16). Short pentraxins can also bind to IgG receptors $(\mathrm{Fc} \gamma \mathrm{R})$. This binding can either inhibit immune 
complex-mediated phagocytosis or activate FcyR-mediated phagocytosis and cytokine secretion (17-20).

\section{The Prototypic Long Pentraxin PTX3}

PTX3 is assembled as multimers of $45 \mathrm{kDa}$ (381 amino acids) subunits and is organized as an octamer with monomers linked by disulfide bounds. Unlike short pentraxins, PTX3 is rapidly neo-synthetized by many cell types $(4,5)$ upon activation by a diversity of signals, including microbes and microbial moieties and inflammatory cytokines $(4,21)$. Contrary to short pentraxins, PTX3 is constitutively expressed, with circulating levels of $\approx 200 \mathrm{ng} / \mathrm{ml}$ in healthy subjects. The levels of PTX3 are strongly and rapidly increased during inflammatory responses $(800-1,000 \mathrm{ng} / \mathrm{ml})$. PTX3 is also preformed in neutrophils and rapidly released upon activation $(22,23)$.

PTX3 binds to the same ligands that short pentraxins, but in a calcium-independent manner (5). Like short pentraxins, PTX3 opsonizes and facilitates the clearance of numerous bacteria (such as Pseudomonas aeruginosa and Salmonella typhimurium) $(24,25)$, virus (such as influenza virus) (26), fungi (such as Aspergillus fumigatus) (27), and apoptotic cells $(4,5)$ by phagocytes. As for CRP and SAP, PTX3 can recruit Fc $\gamma \mathrm{R}$ to induce phagocytosis in a way similar to $\operatorname{IgG}(17,18,20,28,29)$. PTX3 also modulates the complement cascade through its capacity to bind to proteins of the classical (C1q) and lectin (mannose-binding lectin, ficolins) complement pathways (13, 15). PTX 3 thus facilitates the tissue deposition of iC $3 b$, thereby promoting complement receptor 3 (CR3)-driven phagocytosis (28). It also increases the binding of activated complement on microbes initiated by ficolin-1. PTX3 interacts with the complement factor $\mathrm{H}$, the main alternative pathway regulatory protein but without modulating its complement inhibitory activity (30). PTX3 also acts as a nodal point for the assembly of the cumulus oophorus hyaluronan-rich extracellular matrix.

Nevertheless, studies have reported that PTX3 may exhibit a pro-inflammatory activity, underlying its "dual complexity" (31). Indeed, PTX3-deficient mice or mice injected with PTX3 develop less severe phenotypes in various models of sterile inflammation (32-34). Especially, a direct role of PTX3 on endothelial cell functions has been described (i.e., dysfunction and morphological changes in the endothelial layer through a Pselectin/matrix metalloproteinase-1 pathway) $(35,36)$. Considering its diversity of functions, PTX3 is considered as a pivotal sPRM at the crossroad between innate immunity, inflammation, matrix deposition and female fertility (4).

\section{Pentraxins, Cell Death, and Immune Responses}

The management of dying cell clearance is critical to maintain tolerance and to avoid the initiation of auto-immune responses. Schematically, the dichotomy "tolerance versus auto-immunity" is determined by the type of cell death (apoptosis versus necrosis) and the absence or presence of inflammation (either induced by PAMPs or DAMPs) at the time of dying cell clearance.

Apoptosis is characterized by coordinated (programmed) processes allowing dying cells to be eliminated without release of potentially harmful and inflammatory endogenous DAMPs. The clearance of early apoptotic cells is classically viewed as a dynamic tolerogenic process allowing maintaining or inducing regulatory $\mathrm{T}$ cell responses. In contrast, necrosis, which usually occurs following a severe aggression, whether sterile or not, is a passive, accidental cell death process accompanied by the release of intracellular components into the extracellular environment, among which are found potent inflammatory and cytotoxic molecules (such as histones and HMGB1) (37). Importantly, the processing of dying cell-derived antigens in an inflammatory context may lead to the activation/maturation of professional antigen-presenting cells and, ultimately, to the activation of selfreactive $\mathrm{T}$ cells.

Phagocytosis of apoptotic cells, also called efferocytosis, is a fine-tuned process, involving at least three molecular partners (38): (i) eat-me molecules (i.e., neo-expressed motifs allowing discriminating live and dying cells), (ii) sensing and internalization receptors expressed by phagocytes, such as complement component receptor (which recognize collectins), scavenger receptors, integrins, and other receptors (e.g., MER and CR3/CR4), and (iii) sPRM acting as bridging molecules between dying cells and phagocytes.

The pivotal role of opsonins in the clearance of apoptotic cells has been illustrated in C1q- and SAP-deficient mice. C1qdeficient mice spontaneously develop signs of autoimmunity (39) and mice with targeted deletion of the SAP gene spontaneously develop antinuclear autoimmunity and severe glomerulonephritis, a phenotype resembling human SLE (40).

While CRP and SAP have a facilitating role $(11,41,42)$, the role dedicated to PTX3 remains unclear. Indeed, our team has demonstrated that preformed PTX3 contained within neutrophil granules is relocalized at the surface of dying neutrophils, thereby acting as an "eat-me" molecule to mediate their capture by phagocytes (43). In contrast, a study reported that PTX3 binds selectively to late apoptotic cells and inhibits their capture by phagocytes $(42,44)$ suggesting that short and long pentraxins, in the extracellular milieu, may have opposite impacts on apoptotic cells clearance.

It remains also difficult to reconcile in vitro and in vivo data. On the one hand, and contrary to what would have been suspected based on in vitro data, SLE-prone mice supplemented with CRP and CRP-transgenic mice develop a mild kidney disease (45-47). On the other hand, and in agreement with in vitro data, C57/BL6 SAP-deficient mice can produce autoantibodies and develop immune complex glomerulonephritis $(40,48)$. It is important to note that the genetic background is important in enabling autoimmune phenotype expression. Indeed, $\mathrm{SAP}^{-/-} \mathrm{C} 57 / \mathrm{BL} 6$ but not $\mathrm{SAP}^{-/-}$ $129 / \mathrm{Sv}$ mice, spontaneously develop autoantibodies (48). Moreover, the authors also reported that $\mathrm{SAP}^{-1-}$ and $\mathrm{SAP}^{-1-}$ human SAP transgenic mice exhibit a similar autoimmune profile, suggesting that differences may exist between the human and mouse SAP in their capacity to interact with DAMPs. A similar complexity has been reported in C1qdeficient mice, as $\mathrm{C}_{1} \mathrm{q}^{-1-}$ C57/BL6 $6129 \mathrm{~Sv}$ but not $\mathrm{C} \mathrm{q}^{-1-} \mathrm{C} 57 /$ BL6 mice spontaneously develop autoimmunity and 
glomerulonephritis (39). These studies underline the important of genetic background of mice in their susceptibility to develop autoimmunity, suggesting that tolerance breakdown could be strain dependent or dependent on the strategy to knock out target gene expression (impact of genetic recombination on the expression of other key genes) and/or that differences exist between human and mouse sPRM in their capacity to interact with DAMPs. To the best of our knowledge, PTX3-deficient mice do not spontaneously develop signs of autoimmunity. However, they develop a more severe phenotype in a ischemia reperfusion injury model (49) but exhibit a reduced inflammation in an acute arthritis model (50), even though both models are associated with a strong inflammation and, certainly, consecutive or associated to cell death. Moreover, PTX3-deficient mice bred with SLE prone mice have a decreased ability to clear apoptotic cells and tissue damages are aggravated in lung but not in kidney. Collectively, these results suggest that PTX3 deficiency may favor tissue injury associated to the initiation of an autoimmune response (51).

The potential role of pentraxins during auto-immune diseases has been reinforced by translational studies. CRP has been found decreased during SLE flares whereas inflammation markers were elevated $(52,53)$, suggesting that low levels of CRP may be associated with an impaired efferocytosis (54). Moreover, we reported that the levels of PTX3 are lower in patients with SLE during active disease as compared to healthy subjects (55). Whether these decreases impact the efferocytosis process remains undetermined. Nevertheless, these results suggest that a decrease of pentraxin levels which may lead to a reduced efferocytosis, is associated with an autoimmune signature that can be induced by the processing of dying cells in an inflammatory environment.

Collectively, and although the predominant roles of pentraxins seem to be host-protective, detrimental effects were observed in certain experimental settings. This so-called "yinyang" effect may be inherent to the multi-functional properties of pentraxins, the complexity of efferocytosis, the redundancy between sPRM and the potential interaction of pentraxins with different DAMPs released by dying cells (56).

\section{ANTI-PENTRAXIN ANTIBODIES IN AUTOIMMUNE DISEASES}

Antibodies directed against CRP, SAP, and PTX3 have been detected in various autoimmune diseases, especially in SLE and ANCA-associated vasculitis (AAV). Their potential roles in the pathogenesis of autoimmune diseases are described thereafter.

\section{Anti-Pentraxin Autoantibodies in SLE}

SLE is an autoimmune disease with a very heterogeneous clinical presentation. The prognosis is mainly dependent on renal impairment, affecting $50 \%$ to $70 \%$ of patients. SLE is characterized by the production of autoantibodies directed against a large panel of self-antigens, including mainly nuclear antigens (DNA, soluble nuclear antigens $(\mathrm{Sm})$, ribonucleoproteins
(RNP) and histones) but also anti-pentraxin Abs. SLE is also characterized by the activation of complement and the deposition of immune complexes that lead to systemic tissue damages (especially renal, vascular, hematological, cutaneous, and articular lesions) (57). The emergence of autoantibodies is determined by environmental, hormonal, and genetic factors suspected to be involved in the dysregulation of the innate and adaptive immune systems. In a mechanistic point of view, SLE is driven by a strong activation of dendritic cells and the production of type I interferons and is associated to an altered efferocytosis $(57,58)$.

\section{Anti-CRP Antibodies in SLE}

Autoantibodies directed against CRP bind to the monomeric form but not the pentameric form (59). The dissociation of a pentameric to a monomeric form of CRP has been reported in inflammatory situations (60) and monomeric CRP accumulates at the surface of various cell types (B cells, NK cells, platelets) and in inflamed tissues (61).

Anti-CRP Abs have been initially reported in SLE (Table 1) (76). They are present in a significant proportion of patients, ranging from $22 \%$ to $78 \%$. Only one study (65) did not found an increased prevalence of anti-CRP Abs in SLE patients compared to healthy subjects. This conflicting result may be due to different methods of Abs determination.

The correlation between the presence of anti-CRP Abs and clinical presentation at disease onset was not systematically evaluated $(59,62)$. In a study including 137 patients with SLE, Figueredo et al. showed that patients with anti-CRP Abs also had elevated levels of anti-double strand (ds) DNA and antiphospholipids Abs and low levels of the complement component C3. The frequency of anti-CRP Abs was also more frequent in patients with nephritis $(27 \%$ and $13 \%$ in patients with or without nephritis, respectively) (63). Nevertheless, this result was not confirmed in another study (69). In patients with nephritis, anti-CRP Abs were also more frequent (66). Moreover, patients with anti-CRP Abs also had a higher SLEDAI score $(66,70)$ and were more likely to present with acute renal injury (66). However, these results must be nuanced as no relation between disease activity and presence of anti-CRP Abs was found by others $(67,72)$. Focusing on renal involvement, a significant correlation was found between antiCRP Abs and acute renal tubulointerstitial injury (inflammation and fibrosis) but not with specific glomerular lesions (66). Collectively, these data suggest that anti-CRP Abs reflect SLE activity rather than a specific pattern of SLE nephritis.

\section{Anti-SAP Antibodies in SLE}

To the best of our knowledge, only two studies reported the prevalence of anti-SAP Abs in $\operatorname{SLE}(65,73)$ (Table 1). In two studies performed by the same team, Zandman et al (73). included 328 patients with SLE and found anti-SAP Abs in $44 \%$ of patients, slightly above the $20 \%$ found in the second study, as compared with $2 \%$ in healthy subjects (65). As for antiCRP Abs, anti-SAP Abs were not associated with a specific disease phenotype (73), despite an association with anti- 
TABLE 1 | Prevalence of anti-pentraxin antibodies in different autoimmune diseases.

\begin{tabular}{|c|c|c|c|c|c|c|c|}
\hline HS & SLE & PAPS & SSc & ss & RA & AAV & Reference \\
\hline $1 / 40(2.5 \%)$ & 39/50 (78\%) & - & 2/20 (10\%) & - & - & - & (59) \\
\hline $4 / 80(5 \%)$ & 77/137 (51\%) & $68 / 127$ (54\%) & - & - & - & - & (63) \\
\hline - & 43/190 (22.6\%) & - & - & - & 23/103 (22.3\%) & - & (64) \\
\hline $2 / 45$ (4.4\%) & $12 / 150(8 \%)$ & - & - & - & - & - & (65) \\
\hline 0/60 (0\%) & $\begin{array}{l}20 / 49(40.8 \%)^{\star} \\
56 / 96(59.3 \%)^{\star \star}\end{array}$ & - & - & - & - & - & (66) \\
\hline 0/60 (0\%) & - & - & - & 2/7 (28.6\%) & - & $1 / 20(5 \%)$ & (68) \\
\hline- & $26 / 100$ (26\%) & - & - & - & - & - & (69) \\
\hline $21.01 \pm 14.32$ & $35.6 \pm 35.1$ & - & - & - & - & - & (70) \\
\hline $5 / 92(5.4 \%)$ & - & - & - & - & - & $11 / 120$ (9.2\%) & (71) \\
\hline $10 / 36(27.8 \%)$ & 18/34 (52.9\%) & - & - & - & - & - & (72) \\
\hline \multicolumn{8}{|c|}{ Anti-SAP Abs, $n(\%)$} \\
\hline $4 / 93(4.3 \%)$ & $18 / 36(50 \%)$ & - & - & - & $1 / 40(2.5 \%)$ & - & (55) \\
\hline 8/130 (6.2\%) & $60 / 130(46.2 \%)$ & - & $1 / 26(3.8 \%)$ & $2 / 26(7.7 \%)$ & $2 / 27(7.1 \%)$ & - & (74) \\
\hline $12 / 227(5.3 \%)$ & - & - & - & - & - & $56 / 150(37.3 \%)$ & $(71)$ \\
\hline $2 / 100(2 \%)$ & $\begin{array}{c}38 / 196(19.4 \%)^{\star \star} \\
61 / 150(40.7 \%)^{\star}\end{array}$ & - & - & - & - & - & (75) \\
\hline
\end{tabular}

HS, healthy subjects; SLE, systemic lupus erythematosus; PAPS, primary antiphospholipid syndrome; SSc, systemic sclerosis; SS, Sjögren syndrome; RA, rheumatoid arthritis; AAV, ANCA-associated vasculitis.

${ }^{*}$ SLE patients without lupus nephritis.

${ }^{* *}$ SLE patients with lupus nephritis.

dsDNA titers and disease severity index. The levels of auto-Abs lowered under treatment.

\section{Anti-PTX3 Antibodies in SLE}

Anti-PTX3 autoantibodies were first described in SLE patients by our team (55). Their strong prevalence $(\simeq 50 \%$ of patients, as compared to $4 \%$ in healthy subjects) was later confirmed in an independent study (74). In our cohort, we observed an association between anti-PTX3 Ab levels and disease activity (assessed by the SLEDAI score). Antibodies titers were also correlated with classical SLE activity biomarkers, such as antidsDNA Abs, anti-nuclear Abs and $\mathrm{C} 3 / \mathrm{C} 4$ complement components levels (55). However, the study by Bassi et al. did not found any association between anti-PTX3 antibody positivity and SLE activity (74). The authors assessed disease activity using the ECLAM (European Consensus Lupus Activity Measurement) score, making it difficult to compare the two studies. Interestingly, in this study, the authors suggested that anti-PTX3 autoantibodies might provide protection from renal involvement as they represent an independent factor negatively associated with kidney injury. Similar results were reported in a recent study evaluating the prevalence of anti-PTX3 Abs in SLE (75). In this large cohort study, including 246 SLE patients and 100 healthy subjects, Yuan et al. found that patients with SLE nephritis had a lower prevalence of anti-PTX3 Abs compared with patients without nephritis (20\% versus $40 \%$, respectively). Moreover, the serum levels of anti-PTX3 Abs were negatively correlated with proteinuria in lupus nephritis. The levels of proteinuria, serum creatinine and the prevalence of thrombotic microangiopathy were also higher in patients without anti-PTX3 Abs. Although it did not reach statistical significance, patients with anti-PTX3 antibodies were less likely to reach end stage kidney disease. PTX3 deposition and renal fibrosis were also significantly lower in anti-PTX3 Ab-positive patients than negative patients $(74,77)$. These results are consistent with data in animal models. Indeed, lupus prone NZB/NZW mice immunized with PTX3 produce anti-PTX3 Abs and have a delayed occurrence of nephritogenic Abs, a decreased proteinuria, and an increased survival (78).

These results suggest that the balance between the levels of serum PTX3 and anti-PTX3 autoantibodies might influence the nephritic process (progression or attenuation). Anti-PTX3 Abs are not only markers of the inflammatory status in SLE but may also be protective by counteracting the deleterious effects of tissue deposition of PTX3 or complement activation, especially in kidneys. In support, the occurrence of anti-dsDNA and antiC1q Abs in PTX3-immunized mice was significantly delayed and their levels increased when anti-PTX3 autoantibodies were decreasing. Hence, anti-PTX3 antibodies may prevent the proinflammatory deposition of PTX3 in target organs, decreasing the amount of available autoantigens in inflamed kidneys. This suggests that anti-PTX3 Abs might inhibit the secretion of nephritogenic Abs in lupus nephritis (78).

\section{Anti-Pentraxin Antibodies in AAV}

ANCA-associated vasculitis (AAV) include granulomatosis with polyangiitis (GPA), micropolyangiitis (MPA) and granulomatosis with polyangiitis and hypereosinophilia 
(EGPA). AAV are systemic pathologies related to small vessel inflammation whose prognosis is mainly linked to renal (rapidly progressive glomerulonephritis) and pulmonary (intra-alveolar hemorrhage) damages (79). These conditions can rapidly become life-threatening or lead to irreversible functional impairment of the affected organ if they are not promptly treated. Renal or pulmonary impairment can be associated with skin, ear-nose-throat, joint, or neurological damages. These autoimmune diseases are frequently associated with the presence of ANCA, which are diagnostic markers in $80 \%-90 \%$ of patients (80). ANCA-negative vasculitis have been described, especially in patients with EGPA (up to 50\%) but also in patients with GPA or MPA (up to 10\%) (81-83), raising the possibility of other autoantibodies which remain to be identified. The identification of these autoantibodies should be useful for the formal diagnosis of AAV, as early diagnosis is essential to initiate appropriate treatment in order to limit irreversible organ injuries and favor long-term patient survival (84). Some ANCA targeting "minor antigens" have been described (directed against bactericidal/permeability increasing protein, elastase, and cathepsin G) in various autoimmune diseases, especially connective tissue diseases, but their pathogenic role in AAV remains to be demonstrated $(85,86)$. Antibodies against pentraxins have been described in AAV patients.

\section{Anti-CRP Antibodies in AAV}

As reported by our group and by Tan et al. $(68,71)$, anti-CRP Abs were found more frequently in AAV patients as compared to healthy controls, but the levels were similar levels in both groups. To our knowledge, no other study addressed this issue.

\section{Anti-SAP Antibodies in AAV}

In our study, the only one to our knowledge that evaluated the prevalence of anti-SAP Abs in AAV patients (71), anti-SAP Ab titers were higher in AAV patients than in healthy subjects. Moreover, $17.5 \%$ of AAV patients were positive for anti-SAP Abs compared with $3.2 \%$ in the control groups. Among the anti-SAP-positive patients, two were ANCA negative, suggesting that anti-SAP Abs could represent a diagnostic marker in this group of patients.

\section{Anti-PTX3 Antibodies in AAV}

The fact that PTX3 is constitutively expressed by fully differentiated (mature) neutrophils suggests that it may represent a potential target for ANCA. Unlike short pentraxins which are synthetized in the liver (9), PTX3 is stored into neutrophil-specific granules (87). Moreover, PTX3 can be localized in neutrophil extra-cellular traps (NETs) with other intracellular proteins targeted by these autoantibodies, such as MPO and PR3 $(56,88)$. NETs result from a particular type of neutrophil death called NETosis (89), which is characterized by the release of the intracellular content, including genomic DNA. Extracellular DNA thus forms a network to which are associated intracellular molecules such as PR3 and MPO. NETs are microbicide, contributing to fight extracellular infectious agents. However, NETs can also be cytotoxic for endothelial cells and tissues during sterile inflammation. NETosis favors tolerance breakdown, promoting autoimmunity as evidenced by the generation of ANCA through dendritic cell-mediated antigen presentation $(90,91)$. Moreover, ANCA can induce NETs, which can, in turn, activate the complement alternative pathway (9294), leading to an amplification loop of neutrophil activation and death. Indeed, C5a will further attracts and primes neutrophils, rendering them more sensitive to ANCA-mediated activation.

Anti-PTX3 Abs were assessed in the serums of $150 \mathrm{AAV}$ patients by our team (71). Nearly $40 \%$ of AAV patients had antiPTX3 Abs. Moreover, Abs titers were higher in patients with active than in those with inactive AAV. In our cohort, 14 patients (6 EGPA, $6 \mathrm{MPA}$, and $2 \mathrm{GPA}$ ) were positive for indirect immunofluorescence (IIF) ANCA screening, but negative for major (MPO, PR3) and minor (lactoferrin, BPI, elastase, and cathepsin G) ANCA antigens. Interestingly half of them (7/14) had detectable anti-PTX3 Abs. Moreover, anti-PTX3 Abs were found to have a unique IIF pattern known as "small cytoplasmic" ANCA characterized by small-size cytoplasmic granules in methanol- and ethanol-fixed neutrophils, and less intense in formol-fixed neutrophils.

Nevertheless, we did not find any clue for a pathological role of these Abs. Indeed, no difference of renal outcome between anti-PTX3 autoantibody-positive and -negative patients was observed and no relation between anti-PTX3 Ab levels and kidney function at the time of AAV diagnosis was observed (personal unpublished data). It would probably be interesting to evaluate the prevalence of anti-PTX3 Abs in a larger cohort of ANCA-negative patients before to conclude on their clinical relevance and predictive value.

We may wonder whether anti-PTX3 Abs are really ANCA. Indeed, anti-PTX 3 Abs were found positive by immunofluorescence on fixed neutrophils and anti-PTX3 Ab positivity was observed in ANCA-negative AAV patients. Finally, the biological role(s) of antiPTX3 Abs remain(s) to be evaluated. The purification of anti-PTX3 Abs from the serum of patients without other detectable ANCA would allow analyzing the in vitro biological activity of these autoantibodies.

\section{Anti-Pentraxin Antibodies in Other Autoimmune Systemic Diseases Anti-Short Pentraxin Antibodies}

Anti-CRP Abs have been identified in other autoimmune diseases, such as systemic sclerosis (SSc), rheumatoid arthritis (RA), and Sjögren syndrome (SS) patients, although at a lower prevalence than in SLE patients (Table 1). Interestingly, a high prevalence of antiCRP Abs (54\%) has been reported in patients with primary antiphospholipid syndrome (PAPS) (63). Patients with PAPS and antiCRP Abs were more likely to present thrombosis or fetal loss (55\% vs $20 \%$ ). More studies are needed to evaluate if anti-CRP Abs could be used as a biomarker for thrombosis risk assessment.

To the best of our knowledge, the presence of anti-SAP Abs in disease other that SLE or AAV has not been reported.

\section{Anti-PTX3 Antibodies}

Anti-PTX3 Abs have been detected in other autoimmune diseases such as SSc, SS and RA, but at a frequency similar to healthy subjects (74). 


\section{ANTI-PENTRAXIN ANTIBODIES: PATHOGENIC ACTORS OR BYSTANDER MARKERS?}

The presence of anti-pentraxin autoantibodies has been reported mainly in SLE and AAV. Interestingly, antibody levels are generally high at disease onset and decline during remission phases, suggesting that they may represent, at least, diagnostic markers.

It should be noted that, given the strong homology between pentraxins, and in particular between the short pentraxins CRP and SAP, the potential cross-reactivity of autoantibodies directed against pentraxins can be a source of error on the nature of the target as well as on their potential pathological roles.

At this time, we do not know whether these antibodies reflect only tolerance breakdown, like many other autoantibodies reported in these pathologies, or whether they play a pathophysiological role. A potential pathological role for autoantibodies is supported by data on MPO-ANCA for which a pathogenic role is highly suspected. In addition to their capacity to activate neutrophils, MPO-ANCA induce glomerulonephritis when injected in mice (95) and vasculitis in newborns after placental transmission (96). Although neonatal lupus with congenital atrioventricular block after placental transmission anti-Sjögren's-syndrome-related antigen A (SSA) Abs has been described (97), the pathogenicity of antinuclear Abs remains a matter of debate.

To date, no experimental in vitro or in vivo data supports a pathogenic role for anti-pentraxin Abs. Purifying anti-pentraxin Abs may allow to evaluate their potential pathological role, as previously described for anti-MPO and anti-PR3 Abs. However, the cross-reactivity of anti-pentraxin antibodies, which is more than likely due to strong sequence (especially between CRP, SAP, and the C-terminal domain of PTX3) and structure homologies (accessibility to shared conformational epitopes exposed at the surface of pentraxins), makes it difficult to draw a conclusion. Moreover, different epitopes of each pentraxin could be recognized by different antibodies, and the resulting functional outcomes may have different consequences. The isotype of the anti-pentraxin autoantibodies, the site of production (tissue versus mucosal barriers) and the immune status of the tissue at the time of efferocytosis (such as chronic inflammation) can also have important consequences on the regulatory versus activating potential of these autoantibodies. Such complexity could lead to misinterpretation of the roles of anti-PTX Abs in the pathogenesis of autoimmune diseases.

Nevertheless, and based on the role of pentraxins in efferocytosis, it is tempting to speculate that these autoantibodies could alter the efferocytosis process and, consequently, the outcome of immune responses against self-antigens.

On the one hand, anti-pentraxin Abs could inhibit or delay apoptotic cells engulfment by professional antigen-presenting cells, leading to the evolution of dying cells from early to late apoptotic cells. Importantly, late apoptotic/secondary necrotic cells can release potent inflammatory DAMPs that can activate antigen-presenting cells and, consequently, induce the initiation of humoral and cellular specific immune responses. On the other hand, anti-pentraxin Abs may induce the phagocytosis of PTXopsonized dying cells via Fc $\gamma \mathrm{R}$, a process that favors antigenpresenting cell activation and antigen processing that, ultimately, favors the initiation of MHC-I and MHC-II immune responses $(98,99)$. Moreover, while the interaction of apoptotic cells with pentraxins usually has an opsonizing role, a reduced internalization of apoptotic cells by dendritic cells has been reported for PTX3. The authors described this mechanism as "PTX3 censorship", i.e., masking of "eat-me" signals rendering them not detectable by phagocytes (100). Antibodies directed against PTX3 could therefore re-confer immunogenicity to apoptotic cells by promoting the presentation of apoptotic cellassociated antigens by professional APCs and, consequently, the priming and/or expansion of self-reactive T cells. Even though speculative, both mechanisms, which are not exclusive and could occur concomitantly, may favor/maintain the development of autoimmune responses directed against dying cell-associated antigens (Figure 1). Additional studies need to be performed to elucidate the complex dialog between soluble and membrane associated PTX3 (soluble versus) with anti-PTX3 Abs and consequences on sensing and processing of apoptotic cells. Finally, a lower frequency of anti-PTX3 Abs have been reported in patients with lupus nephritis, suggesting that these autoantibodies could also be protecting against PTX3 or complement deposition in kidneys, further enhancing the complexity of the potential pathogenic role of anti-pentraxin auto-antibodies.

Nevertheless, we cannot exclude a protective role for antipentraxin $\mathrm{Ab}$, notably in kidney disorders associated with antipentraxin Abs. Indeed, anti-pentraxin Abs could inhibit the pentraxin deposition in tissue, thereby preventing complement binding and activation. Whether this process may occur in vivo remains undetermined. Another original mechanism has been suggested for anti-SAP Abs. Bickerstaff $M$ et al have reported that SAP inhibits the generation of pathogenic anti-DNA autoantibodies, probably by delaying chromatin degradation (40). This process could be inhibited by anti-SAP Abs, thereby favoring or amplifying the generation of autoantibodies and inflammation.

Nevertheless, it is important to underline that these processes are based on the hypothesis that anti-pentraxin antibodies exhibit mostly similar biological properties. These scenarios can be very different, both in terms of magnitude and nature of the immune response induced, depending on the diversity of autoantibodies produced (isotype, levels) and the presence or absence of each of these pentraxins. The scenario may be even more complicated, particularly due to the involvement of other sPRM and PRR involved in the recognition and removal of danger signals.

\section{CONCLUDING REMARKS}

Pentraxins are soluble PRM involved in antimicrobial defense and efferocytosis. A tolerance breakdown to pentraxins has been 


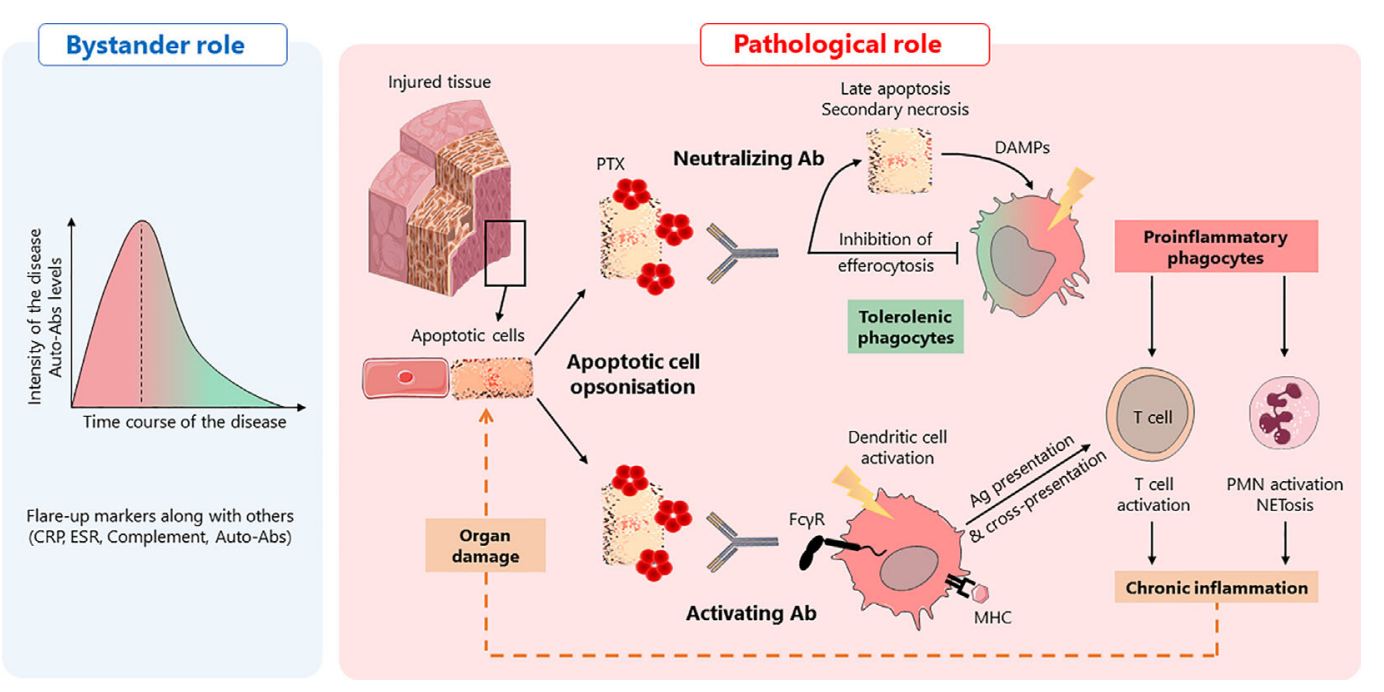

FIGURE 1 | Anti-pentraxin antibodies in autoimmunity: a hypothetical model. Anti-pentraxin antibodies are found elevated at autoimmune disease onset and decline during remission phases, suggesting that they are, at least, diagnostic markers, playing a "bystander role" (left panel). Based on the role of pentraxins in efferocytosis, two hypotheses can be drawn about a hypothetical "pathological role" of anti-pentraxin Abs (right panel). In a physiological context, apoptotic cells are opsonized by pentraxins (PTX) that facilitate their engulfment. This process promotes tolerogenic phagocytes and prevents inflammation. The presence of antipentraxin Abs can lead to the initiation of adaptive immune responses against dying cell antigens and NETosis, a pro-inflammatory process associated with the release of autoantigens. First, anti-PTX Ab may behave as neutralizing antibodies, inhibiting apoptotic cells engulfment by professional antigen-presenting cells, leading to the evolution of dying cells from early to late apoptotic/necrotic cells. These dying cells release potent inflammatory DAMPs inducing the activation of antigen-presenting cells. Second, anti-PTX Ab can be considered as activating Ab, inducing the phagocytosis of dying cells via FcyR. This process also induces the activation of antigen-presenting cells, favoring the initiation of $\mathrm{MHC}-\mathrm{I}$ and $\mathrm{MHC}-\mathrm{II}$ immune responses through antigen presentation and cross-presentation. Whatever the "function" of anti-pentraxin Ab, they initiate an inflammatory process leading to a deleterious amplification loop responsible for tissue damages.

evidenced during various autoimmune diseases. Especially, antiPTX3 autoantibodies are frequent in SLE and AAV and may be of interest for diagnosis, disease activity, response to treatment and outcomes. Moreover, anti-PTX3 Abs share some characteristics with ANCAs and are associated with a specific indirect immunofluorescence neutrophil staining, suggesting that they differ from other ANCA. The prevalence of antiPTX3 Abs appears to be high during ANCA-negative AAV and could constitute a useful marker in these settings. Prospective longitudinal clinical studies are required to confirm the potential of anti-PTX3 Abs as diagnosis and prognosis markers. In a mechanistic point of view, studies are required to determine the potential pathogenic role of anti-PTX3 and whether they should be targeted if they are more than just bystanders in severe autoimmune diseases.

\section{REFERENCES}

1. Janeway CA, Medzhitov R. Innate immune recognition. Annu Rev Immunol (2002) 20:197-216. doi: 10.1146/annurev.immunol.20.083001.084359

2. Locati M, Curtale G, Mantovani A. Diversity, Mechanisms, and Significance of Macrophage Plasticity. Annu Rev Pathol (2020) 15:123-47. doi: 10.1146/ annurev-pathmechdis-012418-012718

3. Jeannin P, Jaillon S, Delneste Y. Pattern recognition receptors in the immune response against dying cells. Curr Opin Immunol (2008) 20:530-7. doi: 10.1016/j.coi.2008.04.013

4. Garlanda C, Bottazzi B, Bastone A, Mantovani A. Pentraxins at the crossroads between innate immunity, inflammation, matrix deposition, and female

\section{AUTHOR CONTRIBUTIONS}

BB wrote the first draft of the manuscript. YD and J-FA provided critical revision of the manuscript. All authors contributed to the article and approved the submitted version.

\section{FUNDING}

This study did not receive any specific grant from funding agencies in the public, commercial, or not-for-profit sectors. This work was realized in the context of (i) the LabEX IGO program (National Research Agency; ANR-11-LABX-0016-01) and (ii) the University Hospital of Angers - University of Angers joint program (project 3I-Impact).

fertility. Annu Rev Immunol (2005) 23:337-66. doi: 10.1146/annurev. immunol.23.021704.115756

5. Deban L, Jaillon S, Garlanda C, Bottazzi B, Mantovani A. Pentraxins in innate immunity: lessons from PTX3. Cell Tissue Res (2011) 343:237-49. doi: 10.1007/s00441-010-1018-0

6. Gewurz H, Zhang XH, Lint TF. Structure and function of the pentraxins. Curr Opin Immunol (1995) 7:54-64. doi: 10.1016/09527915(95)80029-8

7. Guillon C, Bigouagou UM, Folio C, Jeannin P, Delneste Y, Gouet P. A staggered decameric assembly of human C-reactive protein stabilized by zinc ions revealed by X-ray crystallography. Protein Pept Lett (2014) 22:248-55. doi: $10.2174 / 0929866522666141231111226$ 
8. Martinez de la Torre Y, Fabbri M, Jaillon S, Bastone A, Nebuloni M, Vecchi A, et al. Evolution of the pentraxin family: the new entry PTX4. J Immunol Baltim Md 1950 (2010) 184:5055-64. doi: 10.4049/jimmunol.0901672

9. Pepys MB, Hirschfield GM. C-reactive protein: a critical update. J Clin Invest (2003) 111:1805-12. doi: 10.1172/JCI18921

10. Botto M, Hawkins PN, Bickerstaff MC, Herbert J, Bygrave AE, McBride A, et al. Amyloid deposition is delayed in mice with targeted deletion of the serum amyloid P component gene. Nat Med (1997) 3:855-9. doi: 10.1038/ nm0897-855

11. Gershov D, Kim S, Brot N, Elkon KB. C-Reactive protein binds to apoptotic cells, protects the cells from assembly of the terminal complement components, and sustains an antiinflammatory innate immune response: implications for systemic autoimmunity. J Exp Med (2000) 192:1353-64. doi: $10.1084 /$ jem.192.9.1353

12. Agrawal A, Singh PP, Bottazzi B, Garlanda C, Mantovani A. Pattern recognition by pentraxins. Adv Exp Med Biol (2009) 653:98-116. doi: 10.1007/978-1-4419-0901-5_7

13. Inforzato A, Doni A, Barajon I, Leone R, Garlanda C, Bottazzi B, et al. PTX3 as a paradigm for the interaction of pentraxins with the complement system. Semin Immunol (2013) 25:79-85. doi: 10.1016/j.smim.2013.05.002

14. Ng PML, Le Saux A, Lee CM, Tan NS, Lu J, Thiel S, et al. C-reactive protein collaborates with plasma lectins to boost immune response against bacteria. EMBO J (2007) 26:3431-40. doi: 10.1038/sj.emboj.7601762

15. Mantovani A, Valentino S, Gentile S, Inforzato A, Bottazzi B, Garlanda C. The long pentraxin PTX3: a paradigm for humoral pattern recognition molecules. Ann N Y Acad Sci (2013) 1285:1-14. doi: 10.1111/nyas.12043

16. Okemefuna AI, Nan R, Miller A, Gor J, Perkins SJ. Complement factor H binds at two independent sites to C-reactive protein in acute phase concentrations. $J$ Biol Chem (2010) 285:1053-65. doi: 10.1074/jbc.M109.044529

17. Bharadwaj D, Mold C, Markham E, Du Clos TW. Serum amyloid P component binds to $\mathrm{Fc}$ gamma receptors and opsonizes particles for phagocytosis. J Immunol Baltim Md 1950 (2001) 166:6735-41. doi: 10.4049/jimmunol.166.11.6735

18. Mold C, Baca R, Du Clos TW. Serum amyloid P component and C-reactive protein opsonize apoptotic cells for phagocytosis through Fcgamma receptors. J Autoimmun (2002) 19:147-54. doi: 10.1006/jaut.2002.0615

19. Mold C, Gresham HD, Du Clos TW. Serum amyloid P component and Creactive protein mediate phagocytosis through murine $\mathrm{Fc}$ gamma Rs. J Immunol Baltim Md 1950 (2001) 166:1200-5. doi: 10.4049/jimmunol. 166.2.1200

20. Lu J, Marnell LL, Marjon KD, Mold C, Du Clos TW, Sun PD. Structural recognition and functional activation of FcgammaR by innate pentraxins. Nature (2008) 456:989-92. doi: 10.1038/nature07468

21. Basile A, Sica A, d'Aniello E, Breviario F, Garrido G, Castellano M, et al. Characterization of the Promoter for the Human Long Pentraxin PTX3: ROLE OF NF- $\kappa$ B IN TUMOR NECROSIS FACTOR- $\alpha$ AND INTERLEUKIN-1 $\beta$ REGULATION. J Biol Chem (1997) 272:8172-8. doi: $10.1074 /$ jbc.272.13.8172

22. Jaillon S, Peri G, Delneste Y, Frémaux I, Doni A, Moalli F, et al. The humoral pattern recognition receptor PTX3 is stored in neutrophil granules and localizes in extracellular traps. J Exp Med (2007) 204:793-804. doi: 10.1084/ jem.20061301

23. Lominadze G, Powell DW, Luerman GC, Link AJ, Ward RA, McLeish KR. Proteomic analysis of human neutrophil granules. Mol Cell Proteomics MCP (2005) 4:1503-21. doi: 10.1074/mcp.M500143-MCP200

24. Thakur R, Shankar J. In silico Analysis Revealed High-risk Single Nucleotide Polymorphisms in Human Pentraxin-3 Gene and their Impact on Innate Immune Response against Microbial Pathogens. Front Microbiol (2016) 7:192. doi: $10.3389 /$ fmicb. 2016.00192

25. Moalli F, Paroni M, Véliz Rodriguez T, Riva F, Polentarutti N, Bottazzi B, et al. The therapeutic potential of the humoral pattern recognition molecule PTX3 in chronic lung infection caused by Pseudomonas aeruginosa. $J$ Immunol Baltim Md 1950 (2011) 186:5425-34. doi: 10.4049/ jimmunol.1002035

26. Reading PC, Bozza S, Gilbertson B, Tate M, Moretti S, Job ER, et al. Antiviral activity of the long chain pentraxin PTX3 against influenza viruses. J Immunol Baltim Md 1950 (2008) 180:3391-8. doi: 10.4049/ jimmunol.180.5.3391
27. Bozza S, Campo S, Arseni B, Inforzato A, Ragnar L, Bottazzi B, et al. PTX3 binds MD-2 and promotes TRIF-dependent immune protection in aspergillosis. J Immunol Baltim Md 1950 (2014) 193:2340-8. doi: 10.4049/ jimmunol.1400814

28. Moalli F, Doni A, Deban L, Zelante T, Zagarella S, Bottazzi B, et al. Role of complement and $\mathrm{Fc}\{$ gamma\} receptors in the protective activity of the long pentraxin PTX3 against Aspergillus fumigatus. Blood (2010) 116:5170-80. doi: 10.1182/blood-2009-12-258376

29. Mold C, Rodriguez W, Rodic-Polic B, Du Clos TW. C-reactive protein mediates protection from lipopolysaccharide through interactions with $\mathrm{Fc}$ gamma R. J Immunol Baltim Md 1950 (2002) 169:7019-25. doi: 10.4049/ jimmunol.169.12.7019

30. Deban L, Jarva H, Lehtinen MJ, Bottazzi B, Bastone A, Doni A, et al. Binding of the long pentraxin PTX3 to factor $\mathrm{H}$ : interacting domains and function in the regulation of complement activation. J Immunol Baltim Md 1950 (2008) 181:8433-40. doi: 10.4049/jimmunol.181.12.8433

31. Magrini E, Mantovani A, Garlanda C. The Dual Complexity of PTX3 in Health and Disease: A Balancing Act? Trends Mol Med (2016) 22:497-510. doi: 10.1016/j.molmed.2016.04.007

32. Souza DG, Amaral FA, Fagundes CT, Coelho FM, Arantes RME, Sousa LP, et al. The Long Pentraxin PTX3 Is Crucial for Tissue Inflammation after Intestinal Ischemia and Reperfusion in Mice. Am J Pathol (2009) 174:1309_ 18. doi: 10.2353/ajpath.2009.080240

33. Souza DG, Soares AC, Pinho V, Torloni H, Reis LFL, Martins MT, et al. Increased Mortality and Inflammation in Tumor Necrosis Factor-Stimulated Gene-14 Transgenic Mice after Ischemia and Reperfusion Injury. Am J Pathol (2002) 160:1755-65. doi: 10.1016/S0002-9440(10)61122-4

34. Real JM, Spilborghs GMGT, Morato-Marques M, de Moura RP, Negri EM, Camargo AA, et al. Pentraxin 3 accelerates lung injury in high tidal volume ventilation in mice. Mol Immunol (2012) 51:82-90. doi: 10.1016/ j.molimm.2012.02.113

35. Suzuki S, Shishido T, Funayama A, Netsu S, Ishino M, Kitahara T, et al. Long Pentraxin PTX3 Exacerbates Pressure Overload-Induced Left Ventricular Dysfunction. PloS One (2013) 8:e53133. doi: 10.1371/journal.pone.0053133

36. Carrizzo A, Lenzi P, Procaccini C, Damato A, Biagioni F, Ambrosio M, et al. Pentraxin 3 Induces Vascular Endothelial Dysfunction Through a Pselectin/Matrix Metalloproteinase-1 Pathway. Circulation (2015) 131:1495-505. doi: 10.1161/CIRCULATIONAHA.114.014822

37. D'Arcy MS. Cell death: a review of the major forms of apoptosis, necrosis and autophagy. Cell Biol Int (2019) 43:582-92. doi: 10.1002/cbin.11137

38. Morioka S, Maueröder C, Ravichandran KS. Living on the Edge: Efferocytosis at the Interface of Homeostasis and Pathology. Immunity (2019) 50:1149-62. doi: 10.1016/j.immuni.2019.04.018

39. Mitchell DA, Pickering MC, Warren J, Fossati-Jimack L, Cortes-Hernandez J, Cook HT, et al. Clq deficiency and autoimmunity: the effects of genetic background on disease expression. J Immunol Baltim Md 1950 (2002) 168:2538-43. doi: 10.4049/jimmunol.168.5.2538

40. Bickerstaff MCM, Botto M, Hutchinson WL, Herbert J, Tennent GA, Bybee A, et al. Serum amyloid P component controls chromatin degradation and prevents antinuclear autoimmunity. Nat Med (1999) 5:694-7. doi: 10.1038/9544

41. Mihlan M, Stippa S, Józsi M, Zipfel PF. Monomeric CRP contributes to complement control in fluid phase and on cellular surfaces and increases phagocytosis by recruiting factor H. Cell Death Differ (2009) 16:1630-40. doi: 10.1038/cdd.2009.103

42. van Rossum AP, Fazzini F, Limburg PC, Manfredi AA, Rovere-Querini P, Mantovani A, et al. The prototypic tissue pentraxin PTX3, in contrast to the short pentraxin serum amyloid $\mathrm{P}$, inhibits phagocytosis of late apoptotic neutrophils by macrophages. Arthritis Rheum (2004) 50:2667-74. doi: $10.1002 /$ art.20370

43. Jaillon S, Jeannin P, Hamon Y, Frémaux I, Doni A, Bottazzi B, et al. Endogenous PTX3 translocates at the membrane of late apoptotic human neutrophils and is involved in their engulfment by macrophages. Cell Death Differ (2009) 16:465-74. doi: 10.1038/cdd.2008.173

44. Baruah P, Dumitriu IE, Peri G, Russo V, Mantovani A, Manfredi AA, et al. The tissue pentraxin PTX3 limits C1q-mediated complement activation and phagocytosis of apoptotic cells by dendritic cells. J Leukoc Biol (2006) 80:8795. doi: $10.1189 / \mathrm{jlb} .0805445$ 
45. Rodriguez W, Mold C, Marnell LL, Hutt J, Silverman GJ, Tran D, et al. Prevention and reversal of nephritis in MRL/lpr mice with a single injection of C-reactive protein. Arthritis Rheum (2006) 54:325-35. doi: 10.1002/ art.21556

46. Szalai AJ. C-Reactive Protein (CRP) and Autoimmune Disease: Facts and Conjectures. Clin Dev Immunol (2004) 11:221-6. doi: 10.1080/ 17402520400001751

47. Szalai AJ, Weaver CT, McCrory MA, van Ginkel FW, Reiman RM, Kearney $\mathrm{JF}$, et al. Delayed lupus onset in $(\mathrm{NZB} \times \mathrm{NZW}) \mathrm{F}_{1}$ mice expressing a human C-reactive protein transgene: Delayed Lupus Onset in Mice Expressing Human CRP. Arthritis Rheum (2003) 48:1602-11. doi: 10.1002/art.11026

48. Gillmore JD, Hutchinson WL, Herbert J, Bybee A, Mitchell DA, Hasserjian $\mathrm{RP}$, et al. Autoimmunity and glomerulonephritis in mice with targeted deletion of the serum amyloid P component gene: SAP deficiency or strain combination? Immunology (2004) 112:255-64. doi: 10.1111/j.13652567.2004.01860.x

49. de Oliveira THC, Souza DG, Teixeira MM, Amaral FA. Tissue Dependent Role of PTX3 During Ischemia-Reperfusion Injury. Front Immunol (2019) 10:1461. doi: 10.3389/fimmu.2019.01461

50. Batista NV, Barbagallo M, Oliveira VLS, Castro-Gomes T, Oliveira RDR, Louzada-Junior $\mathrm{P}$, et al. The Long Pentraxin 3 Contributes to Joint Inflammation in Gout by Facilitating the Phagocytosis of Monosodium Urate Crystals. J Immunol (2019) 202:1807-14. doi: 10.4049/ jimmunol.1701531

51. Lech M, Römmele C, Kulkarni OP, Susanti HE, Migliorini A, Garlanda C, et al. Lack of the Long Pentraxin PTX3 Promotes Autoimmune Lung Disease but not Glomerulonephritis in Murine Systemic Lupus Erythematosus. PloS One (2011) 6:e20118. doi: 10.1371/journal.pone.0020118

52. Firooz N, Albert D, Wallace D, Ishimori M, Berel D, Weisman M. Highsensitivity C-reactive protein and erythrocyte sedimentation rate in systemic lupus erythematosus. Lupus (2011) 20:588-97. doi: 10.1177/ 0961203310393378

53. Linares LF, Gomez-Reino JJ, Carreira PE, Morillas L, Ibero I. C-reactive protein (CRP) levels in systemic lupus erythematosus (SLE). Clin Rheumatol (1986) 5:66-9. doi: 10.1007/BF02030970

54. Kruse K, Janko C, Urbonaviciute V, Mierke CT, Winkler TH, Voll RE, et al. Inefficient clearance of dying cells in patients with SLE: anti-dsDNA autoantibodies, MFG-E8, HMGB-1 and other players. Apoptosis (2010) 15:1098-113. doi: 10.1007/s10495-010-0478-8

55. Augusto J-F, Onno C, Blanchard S, Dubuquoi S, Mantovani A, Chevailler A, et al. Detection of anti-PTX3 autoantibodies in systemic lupus erythematosus. Rheumatology (2009) 48:442-4. doi: 10.1093/ rheumatology/ken507

56. Daigo K, Hamakubo T. Host-protective effect of circulating pentraxin 3 (PTX3) and complex formation with neutrophil extracellular traps. Front Immunol (2012) 3:378. doi: 10.3389/fimmu.2012.00378

57. Tsokos GC. Systemic Lupus Erythematosus. N Engl J Med (2011) 365:211021. doi: 10.1056/NEJMra1100359

58. Brilland B, Scherlinger M, Khoryati L, Goret J, Duffau P, Lazaro E, et al. Platelets and IgE: shaping the innate immune response in systemic lupus erythematosus. Clin Rev Allergy Immunol (2019) 58(2):194-212. doi: 10.1007/ s12016-019-08744-x

59. Bell SA, Faust H, Schmid A, Meurer M. Autoantibodies to C-reactive protein (CRP) and other acute-phase proteins in systemic autoimmune diseases. Clin Exp Immunol (1998) 113:327-32. doi: 10.1046/j.1365-2249.1998.00655.x

60. Potempa LA, Zeller JM, Fiedel BA, Kinoshita CM, Gewurz H. Stimulation of human neutrophils, monocytes, and platelets by modified C-reactive protein (CRP) expressing a neoantigenic specificity. Inflammation (1988) 12:391405. doi: 10.1007/BF00915774

61. Eisenhardt SU, Thiele JR, Bannasch H, Stark GB, Peter K. C-reactive protein: How conformational changes influence inflammatory properties. Cell Cycle (2009) 8:3885-92. doi: 10.4161/cc.8.23.10068

62. Sjöwall C, Eriksson P, Almer S, Skogh T. Autoantibodies to C-reactive Protein is a Common Finding in SLE, but not in Primary Sjögren's Syndrome, Rheumatoid Arthritis or Inflammatory Bowel Disease. J Autoimmun (2002) 19:155-60. doi: 10.1006/jaut.2002.0608

63. Figueredo MA, Rodriguez A, Ruiz-Yagüe M, Romero M, Fernandez-Cruz A, Gomez-de la Concha E, et al. Autoantibodies against C-reactive protein: clinical associations in systemic lupus erythematosus and primary antiphospholipid syndrome. J Rheumatol (2006) 33:1980-6.

64. Rosenau BJ. Antibodies to C reactive protein. Ann Rheum Dis (2006) 65:674-6. doi: 10.1136/ard.2005.037895

65. Shoenfeld Y, Kravitz MS, Witte T, Doria A, Tsutsumi A, Tatsuya A, et al. Autoantibodies against Protective Molecules C1q, C-Reactive Protein, Serum Amyloid P, Mannose-Binding Lectin, and Apolipoprotein A1: Prevalence in Systemic Lupus Erythematosus. Ann N Y Acad Sci (2007) 1108:227-39. doi: 10.1196/annals.1422.025

66. Tan Y, Yu F, Yang H, Chen M, Fang Q, Zhao M. Autoantibodies against monomeric C-reactive protein in sera from patients with lupus nephritis are associated with disease activity and renal tubulointerstitial lesions. Hum Immunol (2008) 69:840-4. doi: 10.1016/j.humimm.2008.09.006

67. O’Neill SG, Giles I, Lambrianides A, Manson J, D’Cruz D, Schrieber L, et al. Antibodies to apolipoprotein A-I, high-density lipoprotein, and C-reactive protein are associated with disease activity in patients with systemic lupus erythematosus. Arthritis Rheum (2010) 62:845-54. doi: 10.1002/art.27286

68. Tan Y, Yu F, Qu Z, Su T, Xing G-Q, Wu L-H, et al. Modified C-Reactive Protein Might be a Target Autoantigen of TINU Syndrome. Clin J Am Soc Nephrol (2011) 6:93-100. doi: 10.2215/CJN.09051209

69. Pradhan V, Rajadhyaksha A, Yadav K, Surve P, Patwardhan M, Dhavale N, et al. Anti-C reactive protein antibodies in Indian patients with systemic lupus erythematosus. Indian J Nephrol (2013) 23:434. doi: 10.4103/09714065.120341

70. Jung J-Y, Koh B-R, Kim H-A, Jeon J-Y, Suh C-H. Autoantibodies to CReactive Protein in Incomplete Lupus and Systemic Lupus Erythematosus. J Invest Med (2014) 62:890-3. doi: 10.1097/JIM.0000000000000094

71. Simon A, Subra J-F, Guilpain P, Jeannin P, Pignon P, Blanchard S, et al. Detection of Anti-Pentraxin-3 Autoantibodies in ANCA-Associated Vasculitis. PloS One (2016) 11:e0147091. doi: 10.1371/journal.pone.0147091

72. Son C-N, Lee T-H, Bang J-H, Jeong H-J, Chae J-N, Lee W-M, et al. The relationship between anti-C-reactive protein and disease activity in patients with systemic lupus erythematosus. Korean J Intern Med (2018) 33:823-8. doi: $10.3904 / \mathrm{kjim} .2016 .065$

73. Zandman-Goddard G. Anti-serum amyloid component $\mathrm{P}$ antibodies in patients with systemic lupus erythematosus correlate with disease activity. Ann Rheum Dis (2005) 64:1698-702. doi: 10.1136/ard.2005.035832

74. Bassi N, Ghirardello A, Blank M, Zampieri S, Sarzi-Puttini P, Mantovani A, et al. IgG anti-pentraxin 3 antibodies in systemic lupus erythematosus. Ann Rheum Dis (2010) 69:1704-10. doi: 10.1136/ard.2009.117804

75. Yuan M, Tan Y, Pang Y, Li Y, Song Y, Yu F, et al. Anti-pentraxin 3 autoantibodies might be protective in lupus nephritis: a large cohort study. Ren Fail (2017) 39:465-73. doi: 10.1080/0886022X.2017.1308258

76. Robey FA, Jones KD, Steinberg AD. C-reactive protein mediates the solubilization of nuclear DNA by complement in vitro. J Exp Med (1985) 161:1344-56. doi: 10.1084/jem.161.6.1344

77. Bassi N, Del Prete D, Ghirardello A, Gatto M, Ceol M, Zen M, et al. PTX3, Anti-PTX3, and Anti-C1q Autoantibodies in Lupus Glomerulonephritis. Clin Rev Allergy Immunol (2015) 49:217-26. doi: 10.1007/s12016-0158476-9

78. Gatto M, Ghirardello A, Luisetto R, Bassi N, Fedrigo M, Valente M, et al. Immunization with pentraxin 3 (PTX3) leads to anti-PTX3 antibody production and delayed lupus-like nephritis in NZB/NZW F1 mice. J Autoimmun (2016) 74:208-16. doi: 10.1016/j.jaut.2016.07.002

79. Jennette JC, Falk RJ, Bacon PA, Basu N, Cid MC, Ferrario F, et al. revised International Chapel Hill Consensus Conference Nomenclature of Vasculitides. Arthritis Rheum (2013) 65:1-11. doi: 10.1002/art.37715

80. Jennette JC, Nachman PH. ANCA Glomerulonephritis and Vasculitis. Clin J Am Soc Nephrol (2017) 12:1680-91. doi: 10.2215/CJN.02500317

81. Jennette JC, Falk RJ, Hu P, Xiao H. Pathogenesis of Antineutrophil Cytoplasmic Autoantibody-Associated Small-Vessel Vasculitis. Annu Rev Pathol Mech Dis (2013) 8:139-60. doi: 10.1146/annurev-pathol-011811132453

82. Comarmond C, Pagnoux C, Khellaf M, Cordier J-F, Hamidou M, Viallard J-F, et al. Eosinophilic granulomatosis with polyangiitis (Churg-Strauss): Clinical characteristics and long-term followup of the 383 patients enrolled in the French Vasculitis Study Group cohort. Arthritis Rheum (2013) 65:270-81. doi: 10.1002/art.37721 
83. Geetha D, Jefferson JA. ANCA-Associated Vasculitis: Core Curriculum 2020. Am J Kidney Dis (2020) 75:124-37. doi: 10.1053/j.ajkd.2019.04.031

84. Flossmann O, Berden A, de Groot K, Hagen C, Harper L, Heijl C, et al. Longterm patient survival in ANCA-associated vasculitis. Ann Rheum Dis (2011) 70:488-94. doi: 10.1136/ard.2010.137778

85. Beauvillain C, Delneste Y, Renier G, Jeannin P, Subra JF, Chevailler A. Antineutrophil cytoplasmic autoantibodies: how should the biologist manage them? Clin Rev Allergy Immunol (2008) 35:47-58. doi: 10.1007/ s12016-007-8071-9

86. Talor MV, Stone JH, Stebbing J, Barin J, Rose NR, Burek CL. Antibodies to selected minor target antigens in patients with anti-neutrophil cytoplasmic antibodies (ANCA): Minor target antigens in ANCA-associated vasculitis. Clin Exp Immunol (2007) 150:42-8. doi: 10.1111/j.1365-2249.2007.03453.x

87. Jaillon S, Bonavita E, Gentile S, Rubino M, Laface I, Garlanda C, et al. The long pentraxin PTX3 as a key component of humoral innate immunity and a candidate diagnostic for inflammatory diseases. Int Arch Allergy Immunol (2014) 165:165-78. doi: 10.1159/000368778

88. Daigo K, Yamaguchi N, Kawamura T, Matsubara K, Jiang S, Ohashi R, et al. The proteomic profile of circulating pentraxin 3 (PTX3) complex in sepsis demonstrates the interaction with azurocidin 1 and other components of neutrophil extracellular traps. Mol Cell Proteomics (2012) 11:M111.015073. doi: 10.1074/mcp.M111.015073

89. Berthelot J-M, Le Goff B, Neel A, Maugars Y, Hamidou M. NETosis: At the crossroads of rheumatoid arthritis, lupus, and vasculitis. Joint Bone Spine (2017) 84:255-62. doi: 10.1016/j.jbspin.2016.05.013

90. Kessenbrock K, Krumbholz M, Schönermarck U, Back W, Gross WL, Werb $Z$, et al. Netting neutrophils in autoimmune small-vessel vasculitis. Nat Med (2009) 15:623-5. doi: 10.1038/nm.1959

91. Sangaletti S, Tripodo C, Chiodoni C, Guarnotta C, Cappetti B, Casalini P, et al. Neutrophil extracellular traps mediate transfer of cytoplasmic neutrophil antigens to myeloid dendritic cells toward ANCA induction and associated autoimmunity. Blood (2012) 120:3007-18. doi: 10.1182/blood-2012-03416156

92. Söderberg D, Segelmark M. Neutrophil Extracellular Traps in ANCAAssociated Vasculitis. Front Immunol (2016) 7:256. doi: 10.3389/fimmu. 2016.00256

93. Wang H, Wang C, Zhao M-H, Chen M. Neutrophil extracellular traps can activate alternative complement pathways: NETs activate complement in AAV. Clin Exp Immunol (2015) 181:518-27. doi: 10.1111/cei.12654
94. Brilland B, Garnier A-S, Chevailler A, Jeannin P, Subra J-F, Augusto J-F. Complement alternative pathway in ANCA-associated vasculitis: Two decades from bench to bedside. Autoimmun Rev (2020) 19:102424. doi: 10.1016/j.autrev.2019.102424

95. Xiao H, Heeringa P, Hu P, Liu Z, Zhao M, Aratani Y, et al. Antineutrophil cytoplasmic autoantibodies specific for myeloperoxidase cause glomerulonephritis and vasculitis in mice. J Clin Invest (2002) 110:955-63. doi: 10.1172/JCI200215918

96. Schlieben DJ, Korbet SM, Kimura RE, Schwartz MM, Lewis EJ. Pulmonary-renal syndrome in a newborn with placental transmission of ANCAs. Am J Kidney Dis (2005) 45:758-61. doi: 10.1053/j.ajkd. 2005.01.001

97. Brucato A, Frassi M, Franceschini F, Cimaz R, Faden D, Pisoni MP, et al. Risk of congenital complete heart block in newborns of mothers with antiRo/SSA antibodies detected by counterimmunoelectrophoresis: a prospective study of 100 women. Arthritis Rheum (2001) 44:1832-5. doi: 10.1002/1529-0131(200108)44:8<1832::AID-ART320>3.0.CO;2-C

98. Mantegazza AR, Magalhaes JG, Amigorena S, Marks MS. Presentation of phagocytosed antigens by MHC class I and II. Traffic Cph Den (2013) 14:135-52. doi: 10.1111/tra.12026

99. Junker F, Gordon J, Qureshi O. Fc Gamma Receptors and Their Role in Antigen Uptake, Presentation, and T Cell Activation. Front Immunol (2020) 11:1393. doi: 10.3389/fimmu.2020.01393

100. Rovere P, Peri G, Fazzini F, Bottazzi B, Doni A, Bondanza A, et al. The long pentraxin PTX3 binds to apoptotic cells and regulates their clearance by antigen-presenting dendritic cells. Blood (2000) 96:4300-6. doi: 10.1182/ blood.V96.13.4300.h8004300_4300_4306

Conflict of Interest: The authors declare that the research was conducted in the absence of any commercial or financial relationships that could be construed as a potential conflict of interest.

Copyright $\odot 2021$ Brilland, Vinatier, Subra, Jeannin, Augusto and Delneste. This is an open-access article distributed under the terms of the Creative Commons Attribution License (CC BY). The use, distribution or reproduction in other forums is permitted, provided the original author(s) and the copyright owner(s) are credited and that the original publication in this journal is cited, in accordance with accepted academic practice. No use, distribution or reproduction is permitted which does not comply with these terms. 